\title{
Even Set Systems
}

Andreas Dress $1,2 *$

${ }^{1}$ Department for Combinatorics and Geometry, CAS-MPG Partner Institute and Key Lab for Computational Biology, Shanghai Institutes for Biological Sciences, Chinese Academy of Sciences, 320 Yue Yang Road, Shanghai 200031, P.R.China

${ }^{2}$ Max Planck Institute for Mathematics in the Sciences, Inselstrasse 22 -26, D 04103 Leipzig, Germany

dress@ sibs.ac.cn,dress@mis.mpg.de

Received December 3, 2005

AMS Subject Classification: 92B10, 05E99, 05A18

\begin{abstract}
In phylogenetic combinatorics, the analysis of split systems is a fundamental issue. Here, we observe that there is a canonical one-to-one correspondence between split systems on the one, and "even" set systems on the other hand, i.e., given any finite set $X$, we show that there is a canonical one-to-one correspondence between the set $\mathcal{P}(S(X))$ consisting of all subsets $\mathcal{S}$ of the set $\mathcal{S}(X)$ of all splits of the set $X$ (that is, all 2-subsets $\{A, B\}$ of the power set $\mathcal{P}(X)$ of $X$ for which $A \cup B=X$ and $A \cap B=\emptyset$ hold) and the set $\mathcal{P}^{e v e n}(\mathcal{P}(X))$ consisting of all subsets $\mathcal{E}$ of the power set $\mathcal{P}(X)$ of $X$ for which, for each subset $Y$ of $X$, the number of proper subsets of $Y$ contained in $\mathcal{E}$ is even.
\end{abstract}

Keywords: splits, split systems, set systems, combinatorics of split systems, combinatorics of set systems, even set systems, phylogenetic analysis, phylogenetic combinatorics

\section{Introduction}

An important topic in phylogenetic combinatorics is the analysis of split systems, i.e., of subsets $S$ of the set

$$
\mathcal{S}(X):=\{\{A, B\}: A, B \subseteq X, A \cup B=X, A \cap B=\emptyset\}
$$

consisting of all splits $S=\{A, B\}$ of a given finite set $X$, that is, all 2-subsets $\{A, B\}$ of the power set $\mathcal{P}(X)$ of $X$ for which $A \cup B=X$ and $A \cap B=\emptyset$ hold.

Here, we want to present a result that apparently, in spite of all the efforts that has gone into analyzing all sorts of split systems in recent years (see for instance, [1-8]), has gone unnoticed so far, viz., the fact that, given any finite set $X$, there is a canonical one-to-one correspondence between the set $P(S(X))$ consisting of all subsets $\mathcal{S}$ of the

\footnotetext{
* Partly supported by the Science Technology Commission of Shanghai Municipality (Grant 06ZR14048).
} 
set $\mathcal{S}(X)$ and the set $\mathcal{P}^{\text {even }}(\mathcal{P}(X))$ consisting of all even set systems $\mathcal{E}$ defined over $X$ provided we define a subset $\mathcal{E}$ of the power set $\mathcal{P}(X)$ of $X$ to be an even set system (defined over $X$ ) if and only if the number $\mathcal{E}^{*}(Y)$ of proper subsets of any given subset $Y$ of $X$ that are contained in $\mathcal{E}$ is even, that is, if and only if

$$
\mathcal{E}^{*}(Y):=|\{Z \subsetneq Y: Z \in \mathcal{E}\}| \equiv 0 \bmod 2
$$

holds for every subset $Y$ of $X$.

More precisely, we want to establish the following two simple facts:

Proposition 1.1. Given any finite set $X$, any set system $X \subset \mathcal{P}(X)$ defined over $X$, any subset $Y$ of $X$, and any split $S=\{A, B\} \in \mathcal{S}(X)$, put

$$
X(Y):=|\{Z \in X: Y \subseteq Z\}|
$$

and

$$
\mathcal{X}(S):=X(A) \mathcal{X}(B)
$$

Then,

$$
\mathcal{E}(A) \equiv \mathcal{E}(B) \quad \bmod 2
$$

and, therefore, also

$$
\mathcal{E}(S)=\mathcal{E}(A) \mathcal{E}(B) \equiv \mathcal{E}(A) \equiv \mathcal{E}(B) \quad \bmod 2
$$

holds for every even set system $\mathcal{E} \in \mathcal{P}^{\text {even }}(\mathcal{P}(X))$ defined over $X$ and every split $S=$ $\{A, B\} \in \mathcal{S}(X)$ of $X$.

Proposition 1.2. Given any finite set $X$ and any set system $X \subset \mathcal{P}(X)$ defined over $X$, put

$$
\mathcal{S}_{X}:=\{S \in \mathcal{S}(X): X(S) \equiv 1 \bmod 2\} .
$$

Then, restricting the map

$$
\mathcal{P}(\mathcal{P}(X)) \rightarrow \mathcal{P}(\mathcal{S}(X)): X \mapsto \mathcal{S}_{X}
$$

to the subset $\mathcal{P}^{\text {even }}(\mathcal{P}(X))$ of $\mathcal{P}(\mathcal{P}(X))$ consisting of all even set systems $\mathcal{E}$ defined over $X$ induces a canonical bijection from $\mathcal{P}^{\text {even }}(\mathcal{P}(X))$ onto the set $\mathcal{P}(\mathcal{S}(X))$ consisting of all split systems $\mathcal{S}$ defined over $X$ whose inverse is given by associating, to any split system $S \in S(X)$ defined over $X$, the set system

$$
\mathcal{E}_{\mathcal{S}}:=\{Y \subseteq X:|\{Z \subseteq X: Y \subseteq Z,\{Z, X-Z\} \in \mathcal{S}\}| \equiv 1 \bmod 2\} .
$$

\section{Proofs}

Both results follow easily from combining the fact that, given any two finite sets $Y, Z$ with $Y \subseteq Z$, one has

$$
|\{A \subseteq Z: Y \subseteq A\}| \equiv 1 \bmod 2
$$


if and only if $Y=Z$ holds, with the fact that, putting

$$
\delta_{Y, Z}:= \begin{cases}1, & \text { if } Y=Z \\ 0, & \text { else }\end{cases}
$$

for all $Y, Z \subseteq X$ (as usual), and

$$
\delta_{A, X}:=\sum_{Y \in X} \delta_{A, Y}= \begin{cases}1, & \text { if } A \in X \\ 0, & \text { else }\end{cases}
$$

for every set system $X \subseteq \mathcal{P}(X)$ defined over $X$ and all $A \subseteq X$ (also as usual), one has

$$
\sum_{Y \subseteq Z} \delta_{Y, \mathcal{E}}=|\{Y \in \mathcal{E}: Y \subseteq Z\}|=\mathcal{E}^{*}(Z)+\delta_{Z, \mathcal{E}} \equiv \delta_{Z, \mathcal{E}} \quad \bmod 2
$$

for every even set system $\mathcal{E} \in \mathcal{P}^{\text {even }}(\mathcal{P}(X))$ defined over $X$ and every subset $Y$ of $X$ and, therefore,

$$
\mathcal{E}(A)=\sum_{A \subseteq Z \subseteq X} \delta_{Z, \mathcal{E}} \equiv \sum_{A \subseteq Z \subseteq X}|\{Y \in \mathcal{E}: Y \subseteq Z\}| \bmod 2
$$

for every even set system $\mathcal{E} \in \mathcal{P}^{\text {even }}(\mathcal{P}(X))$ defined over $X$ and every subset $A$ of $X$.

Indeed, given any finite set $X$, any even set system $\mathcal{E} \in \mathcal{P}^{\text {even }}(\mathcal{P}(X))$ defined over $X$, and any split $S=\{A, B\} \in \mathcal{S}(X)$ of $X$, the above formulae imply that

$$
\begin{aligned}
\mathcal{E}(A) & =\sum_{A \subseteq Z \subseteq X} \delta_{Z, \mathcal{E}} \\
& \equiv \sum_{A \subseteq Z \subseteq X}|\{Y \in \mathcal{E}: Y \subseteq Z\}| \quad \bmod 2 \\
& =\sum_{A \subseteq Z \subseteq X} \sum_{Y \subseteq Z} \delta_{Y, \mathcal{E}} \\
& =\sum_{Y \subseteq X} \delta_{Y, \mathcal{E}}|\{Z \subseteq X: A \cup Y \subseteq Z\}| \\
& \equiv \sum_{Y \subseteq X} \delta_{Y, \mathcal{E}} \delta_{A \cup Y, X} \quad \bmod 2 \\
& =\sum_{B \subseteq Y \subseteq X} \delta_{Y, \mathcal{E}} \\
& =\mathcal{E}(B)
\end{aligned}
$$

holds. This establishes the first proposition.

To show that also the second proposition holds, we have to show that

(i) $\mathcal{E}_{\mathcal{S}_{\mathcal{E}}}=\mathcal{E}$ holds for every even set system $\mathcal{E} \in \mathcal{P}^{\text {even }}(\mathcal{P}(X))$,

(ii) $\mathcal{E}_{\mathcal{S}}$ is an even set system for every split system $\mathcal{S} \subseteq \mathcal{S}(X)$,

(iii) $\mathcal{S}_{\mathcal{E}_{\mathcal{S}}}=S$ holds for all $S \subseteq S(X)$. 
So, assume that $\mathcal{E} \in \mathcal{P}^{\text {even }}(\mathcal{P}(X))$ is an even set system and that $Y$ is any subset of $X$. Then, $Y$ is contained in $\mathcal{E}_{\mathcal{S}_{\mathcal{E}}}$ if and only if

$$
\left|\left\{Z \subseteq X: Y \subseteq Z,\{Z, X-Z\} \in \mathcal{S}_{\mathcal{E}}\right\}\right| \equiv 1 \bmod 2
$$

or, equivalently,

$$
|\{Z \subseteq X: Y \subseteq Z, \mathcal{E}(Z) \equiv 1 \bmod 2\}| \equiv 1 \bmod 2
$$

holds. However, we have

$$
\begin{aligned}
\mid\{Z \subseteq X: Y \subseteq Z, \mathcal{E}(Z) & \equiv 1 \bmod 2\} \mid \equiv \sum_{Z \subseteq X, Y \subseteq Z} \mathcal{E}(Z) \bmod 2, \\
\sum_{Z \subseteq X, Y \subseteq Z} \mathcal{E}(Z) & =\sum_{Z \subseteq X, Y \subseteq Z} \sum_{W \subseteq X, Z \subseteq W} \delta_{W, \mathcal{E}} \\
& =\sum_{W \subseteq X, Y \subseteq W} \sum_{Z \subseteq W, Y \subseteq Z} \delta_{W, \mathcal{E}} \\
& =\sum_{W \subseteq X, Y \subseteq W} 2^{|W-Y|} \delta_{W, \mathcal{E}},
\end{aligned}
$$

and

$$
\sum_{W \subseteq X, Y \subseteq W} 2^{|W-Y|} \delta_{W, \mathcal{E}} \equiv \sum_{W \subseteq X, Y \subseteq W} \delta_{Y, W} \delta_{W, \mathcal{E}}=\delta_{Y, \mathcal{E}} \quad \bmod 2
$$

therefore,

$$
Y \in \mathcal{E}_{\mathcal{S}_{\mathcal{E}}} \Longleftrightarrow Y \in \mathcal{E}
$$

for every $Y \subseteq X$ implying that $\mathcal{E}=\mathcal{E}_{\mathcal{S}_{\mathcal{E}}}$ holds indeed for every even set $\mathcal{E} \subseteq \mathcal{P}(X)$, as claimed.

Next, given any split system $\mathcal{S} \subseteq \mathcal{S}(X)$, note first that

$$
\delta_{Y, \mathcal{E}_{\mathcal{S}}} \equiv \sum_{Z \subseteq X, Y \subseteq Z} \delta_{\{Z, X-Z\}, \mathcal{S},} \bmod 2
$$

as well as

$$
\mathcal{E}_{\mathcal{S}}(Y)=\left|\left\{Z \in \mathcal{E}_{\mathcal{S}}: Y \subseteq Z\right\}\right|=\sum_{Z \subseteq X, Y \subseteq Z} \delta_{Z, \mathcal{E}_{\mathcal{S}}}
$$

holds, essentially by definition, for every subset $Y$ of $X$ (with $\delta_{S, \mathcal{S}}:=1$ if $S \in \mathcal{S}$ holds, 
and $\delta_{S, \mathcal{S}}:=0$ if this does not hold, of course) implying that

$$
\begin{aligned}
\sum_{Z \subseteq Y} \delta_{Z, \mathcal{E}_{S}} & \equiv \sum_{Z \subseteq Y} \sum_{W \subseteq X, Z \subseteq W} \delta_{\{W, X-W\}, \mathcal{S}} \bmod 2 \\
& \equiv \sum_{W \subseteq X} \sum_{Z \subseteq Y \cap W} \delta_{\{W, X-W\}, S} \bmod 2 \\
& \equiv \sum_{W \subseteq X} 2^{|Y \cap W|} \delta_{\{W, X-W\}, \mathcal{S}} \bmod 2 \\
& \equiv \sum_{W \subseteq X-Y} \delta_{\{W, X-W\}, S} \bmod 2 \\
& \equiv \sum_{W: Y \subseteq X-W} \delta_{\{W, X-W\}, \mathcal{S}} \bmod 2 \\
& \equiv \sum_{W: Y \subseteq W} \delta_{\{W, X-W\}, \mathcal{S}} \\
& \equiv \delta_{Y, \mathcal{E}_{\mathcal{S}}} \bmod 2
\end{aligned}
$$

and, therefore,

$$
\mathcal{E}_{S}^{*}(Y)=\sum_{Z \subsetneq Y} \delta_{Z, \mathcal{E}_{S}}=\sum_{Z \subseteq Y} \delta_{Z, \mathcal{E}_{\mathcal{S}}}-\delta_{Y, \mathcal{E}_{\mathcal{S}}} \equiv 0 \quad \bmod 2
$$

holds also for every subset $Y$ of $X$, as claimed.

Consequently, one has

$$
\mathcal{E}_{\mathcal{S}}(S) \equiv \mathcal{E}_{\mathcal{S}}(A) \equiv \mathcal{E}_{\mathcal{S}}(B) \quad \bmod 2
$$

for every split system $\mathcal{S} \subseteq \mathcal{S}(X)$ and every split $S=\{A, B\} \in \mathcal{S}(X)$. Thus, given a split $S=\{A, B\} \in \mathcal{S}(X)$ and a split system $S \subseteq S(X)$, one has $S \in \mathcal{S}_{\mathcal{E}_{\mathcal{S}}}$ if and only if one has $\mathcal{E}_{S}(A) \equiv 1 \bmod 2$ holds. However, one has

$$
\mathcal{E}_{\mathcal{S}}(Y)=\left|\left\{Z \in \mathcal{E}_{\mathcal{S}}: Y \subseteq Z\right\}\right|=\sum_{Z \subseteq X, Y \subseteq Z} \delta_{Z, \mathcal{E}_{\mathcal{S}}}
$$

as well as

$$
\delta_{Y, \mathcal{E}_{\mathcal{S}}} \equiv \sum_{Z \subseteq X, Y \subseteq Z} \delta_{\{Z, X-Z\}, \mathcal{S}, \quad \bmod 2}
$$

essentially by definition, for every subset $Y$ of $X$ implying that

$$
\begin{aligned}
\mathcal{E}_{S}(A) & =\sum_{Z \subseteq X, A \subseteq Z} \delta_{Z, \mathcal{E}_{S}} \\
& \equiv \sum_{Z \subseteq X, A \subseteq Z W \subseteq X, Z \subseteq W} \sum_{\{W, X-W\}, S} \delta_{W} \bmod 2 \\
& \equiv \sum_{W \subseteq X} \sum_{A \subseteq Z \subseteq W} \delta_{\{W, X-W\}, S} \bmod 2
\end{aligned}
$$




$$
\begin{aligned}
& \equiv \sum_{W \subseteq X} 2^{|W-A|} \delta_{\{W, X-W\}, \mathcal{S}} \bmod 2 \\
& \equiv \delta_{\{A, X-A\}, \mathcal{S}} \\
& =\delta_{\{A, B\}, \mathcal{S}} \\
& =\delta_{S, \mathcal{S}} \quad \bmod 2
\end{aligned}
$$

holds for every split $S=\{A, B\} \in \mathcal{S}(X)$ and, therefore, also

$$
S \in \mathcal{S}_{\mathcal{E}_{\mathcal{S}}} \Longleftrightarrow \mathcal{E}_{S}(A) \equiv 1 \bmod 2 \Longleftrightarrow \delta_{S, S}=1 \Longleftrightarrow S \in \mathcal{S} .
$$

Thus, we must have $\mathcal{S}_{\mathcal{E}_{\mathcal{S}}}=\mathcal{S}$ for every split system $\mathcal{S} \subseteq \mathcal{S}(X)$, as required.

\section{References}

1. J. Backelin and S. Linusson, Parity splits by triple point distances in $X$-trees, Ann. Combin. 10 (1) (2006) 1-18.

2. H.-J. Bandelt and A. Dress, A canonical decomposition theory for metrics on a finite set, Adv. Math. 92 (1) (1992) 47-105.

3. H.-J. Bandelt and A. Dress, Split decomposition: a new and useful approach to phylogenetic analysis of distance data, Mol. Phylogenet Evol. 1 (3) (1992) 242-252.

4. A. Dress and M.A. Steel, Phylogenetic diversity over an abelian group, Ann. Combin. 11 (2) (2007) 143-160.

5. A.W.M. Dress, Split decomposition over an abelian group, part 1: generalities, Ann. Combin. 13 (2009) 199-232.

6. A.W.M. Dress, Split decomposition over an abelian group, part 2: group-valued split systems with weakly compatible support, Discrete Appl. Math. 157 (10) 2349-2360.

7. A.W.M. Dress, Split decomposition over an abelian group, part 3: group-valued split systems with compatible support, Shanghai, manuscript.

8. C. Semple and M.A. Steel, Phylogenetics, Oxford University Press, Oxford, 2003.

Open Access This article is distributed under the terms of the Creative Commons Attribution Noncommercial License which permits any noncommercial use, distribution, and reproduction in any medium, provided the original author(s) and source are credited. 